\title{
A REMARK ABOUT HOMOGENEOUS POLYNOMIAL MAPS
}

\author{
Aleksey Tret'yakov - Henryk ŻoŁąDek
}

\begin{abstract}
We consider homogeneous polynomial maps $F: \mathbb{R}^{n} \rightarrow \mathbb{R}^{n}$ of degree $p$. We classify the pairs $(p, n)$ for which there exists a surjective and non-proper such map and when the right inverse to $F$ exists but is unbounded.
\end{abstract}

\section{Introduction}

In this work we study maps $F: X \mapsto Z$, where $X=Z=\mathbb{R}^{n}$ and $F$ is a homogeneous polynomial map (of degree $p \geq 1$ ), i.e. $F(\lambda x)=\lambda^{p} F(x)$.

For such maps one defines their kernel

$$
\operatorname{ker}^{p} F=\left\{x \in \mathbb{R}^{n}: F(x)=0\right\} .
$$

Note that $\operatorname{ker}^{p} F$ is a subset of $X=\mathbb{R}^{n}$ invariant with respect to the action of $\mathbb{R}^{*}=\mathbb{R} \backslash 0, x \mapsto \lambda x$

In the case of homogeneous linear maps $L: \mathbb{R}^{n} \mapsto \mathbb{R}^{n}$ the theorem about rank states that: $L$ is injective if and only if it is surjective, i.e. $\operatorname{ker} L=0 \Leftrightarrow$ $\operatorname{Im} L=\mathbb{R}^{n}$. In that case the map $L$ is invertible and the inverse map is bounded, $\left\|L^{-1}\right\|<\infty$. This property is important in the nonlinear analysis, when one solves nonlinear equations using linear approximations (the Newton method).

2000 Mathematics Subject Classification. Primary 47H60, 58C05; Secondary 57R25. field.

Key words and phrases. Homogeneous polynomial map, index of singular point of vector

Supported by Russian Found of Fundamental Research No 96-01-00288, 97-01-00188.

Supported by Polish KBN Grant No 2/P03A/010/22. 
Recently we observe some progress in generalization of the computational methods to the singular cases (see [3]), e.g. when the solution lies in the set of critical points. For such equations $G(x)=0$ with a solution $x_{0}$ such that $G_{*}\left(x_{0}\right)=0$ the first nonzero homogeneous part $F=(1 / p !) G_{p}$ of the Taylor expansion $\sum(1 / m !) G_{m}\left(x-x_{0}\right)$ is important, it plays a role of linear approximation.

One is interested in existence of the right inverse $H \stackrel{\mathrm{df}}{=}\left\{F^{-1}\right\}_{r}: Z \mapsto X$ to $F$, i.e. such that $F \circ H=$ id. The map $H$ exists when $F$ is surjective, but it can be not unique and not continuous; it is composed of branches of an algebraic multivalued map.

From the point of view of applications (e.g. in nonlinear analysis) not only the existence of the right inverse $H=\left\{F^{-1}\right\}_{r}$ to $F$ is important. Maybe even more important is its boundedness, i.e. we ask whether one can choose $H$ in such a way that $|H(z)|<C$ if $|z| \leq 1$ for some constant $C$. Such property is called the Banach property of $F$ (see [9], [3], [4]). In [3] there is an example of homogeneous quintic map of a plane for which $\left\{F^{-1}\right\}_{r}$ exists (i.e. $F$ is surjective), but it cannot be chosen as bounded.

Recall that by definition a continuous map $F: X \mapsto Z$ is proper if preimage of any compact subset of $Z$ is a compact subset of $X$.

We have the following characterization of proper homogeneous polynomial maps.

Lemma 1. Let $F$ be as above. Then $F$ is proper if and only if $\operatorname{ker}^{p} F=0$. Moreover, if $F$ is proper and surjective, then any right inverse to $F$ is bounded.

Proof. If $\operatorname{ker}^{p} F \neq 0$ and $x_{0} \in \operatorname{ker}^{p} F \backslash 0$, then the whole unbounded line $\mathbb{R} x_{0}$ is sent to 0 . So the preimage of 0 cannot be compact.

Assume that $\operatorname{ker}^{p} F=0$. Let $\mathbb{S}=\{x \in X:|x|=1\}$ be the standard unit sphere. No point of $\mathbb{S}$ is sent to 0 , so $0 \notin F(\mathbb{S})$. Of course, $F(\mathbb{S})$ is compact. Hence the point $0 \in Z$ is separated from $F(\mathbb{S})$, i.e. $\inf _{z \in F(\mathbb{S})}|z|=A>0$.

Any $x \in X \backslash 0$ can be written in the form $x=r x_{0}$, where $r=|x|>0$ and $x_{0} \in \mathbb{S}$. We have then $F(x)=r^{p} F\left(x_{0}\right)$ and hence $|F(x)| \geq r^{p} A=A|x|^{p}$. We see that $|F(x)| \rightarrow \infty$ as $|x| \rightarrow \infty$.

This is equivalent to the properness of $F$.

For any continuous map $F: X \mapsto Z$ one defines the non-properness set $S(F) \subset$ $Z$ as consisting of those points $z_{0}$ which have neighbourhoods with compact closures whose inverse images are unbounded. In other words, $z_{0} \in S(F)$ if and only if there exists a sequence $z_{n} \rightarrow z_{0}$ admitting preimages $x_{n} \in F^{-1}\left(z_{n}\right)$ forming an unbounded sequence, $\left|x_{n}\right| \rightarrow \infty$. The map $F$ is proper if and only if $S(F)=\emptyset$.

Assume that $F$ is proper and surjective and suppose that some its right inverse $H$ is unbounded. Then there should exist a sequence $z_{n} \in Z$ with norms 
bounded by 1 such that $x_{n} \stackrel{\text { df }}{=} H\left(z_{n}\right) \rightarrow \infty$ (i.e. is unbounded). Eventually passing to a subsequence, we can assume that the sequence $\left\{z_{n}\right\}$ is convergent to some $z_{0}$. But this would imply that $z_{0} \in S(F)$, in contradiction to the assumption about properness of $F$.

This gives the second statement of Lemma 1.

One cannot expect equivalence of the properties $\operatorname{ker}^{p} F=0$ and $\operatorname{Im} F=\mathbb{R}^{n}$. Indeed, the one-dimensional maps $F(x)=x^{2 k}$ have $\operatorname{ker}^{2 k} F=0$, but $\operatorname{Im} F=$ $\{z \geq 0\}$. However, the implication

$$
\operatorname{ker}^{p} F \neq 0 \Rightarrow \operatorname{Im} F \neq \mathbb{R}^{n}
$$

for quadratic homogeneous maps $F: \mathbb{R}^{n} \mapsto \mathbb{R}^{n}$ seems quite possible.

A. Izmailov and A. Tret'yakov ([5]) have proved that when $n=1,2,3$ and $p=2$ the implication (1.1) holds true. (We refer the reader to [6] for geometry of quadratic maps). The first aim of the present work is to solve completely the problem of maps which contradict the implication (1.1).

\section{Theorem 1.}

(a) Assume that either $p \geq 3, n \geq 2$, or $p=2$, $n \geq 4$. Then there exists a polynomial homogeneous map $\mathbb{R}^{n} \mapsto \mathbb{R}^{n}$ of degree $p$ which is surjective and not proper.

(b) Assume that either $p=1$, or $n=1$, or $p=2, n=2,3$. Then any such map satisfies (1.1).

Our second result concerns the question of existence of bounded right inverses to surjective maps.

\section{Theorem 2.}

(a) If $p \geq 4, n \geq 2$, or $p=3, n \geq 3$ then there exist examples of surjective maps for which any right inverse map is unbounded.

(b) If $p=1$, or $n=1$, or $p=2, n=2,3$, or $p=3, n=2$ then any surjective map admits a bounded right inverse.

We see that the cases $p=2, n \geq 4$ are unsolved. The examples of non-proper and surjective quadratic maps from Theorem 1(a) have bounded right inverses.

\section{Examples of non-proper and surjective maps}

In all the examples below we have $X=\tilde{X} \times \tilde{Y}=\{(x, y)\}$, where $\tilde{X}=\mathbb{R}^{n-k}$, $\tilde{Y}=\mathbb{R}^{k}, 1<k<n$ and the map $F$ is such that $F(0, y)=0$. Thus $\{0\} \times \tilde{Y} \subset$ $\operatorname{ker}^{p} F$ and $F$ is not proper. 
2.1. The case $p \geq 3$ odd and $n \geq 2$. We represent the source space $\mathbb{R}^{n}$ as $\mathbb{R}^{n-1} \times \mathbb{R}^{1}=\{(x, y)\}=\left\{\left(x_{1}, \ldots, x_{n-1}, y\right)\right\}$. We define the map $F$ as follows

$$
(x, y) \mapsto\left(x_{1}^{p}, \ldots, x_{n-2}^{p}, x_{n-1}^{p-1} y, x_{n-1}^{p-2}\left(y^{2}-x_{n-1}^{2}\right)\right) .
$$

We see that the line $\{0\} \times \mathbb{R}^{1}=\{x=0\}$ is mapped to 0 . Therefore it remains to show that it is surjective, i.e. that for any $\left(z_{1}, \ldots, z_{n}\right)$ the system of equations

$$
\begin{aligned}
x_{1}^{p} & =z_{1}, \\
\ldots \ldots & \ldots \ldots \\
x_{n-2}^{p} & =z_{n-2}, \\
x_{n-1}^{p-1} y & =z_{n-1}, \\
x_{n-1}^{p-2}\left(y^{2}-x_{n-1}^{2}\right) & =z_{n}
\end{aligned}
$$

has a solution.

Since $p$ is odd, we easily solve the first $n-2$ equations. Thus the problem is reduced to the problem of solution of the system

$$
x^{p-1} y=z, \quad x^{p-2}\left(y^{2}-x^{2}\right)=u .
$$

If $z=0$, then we get $y=0, x=\sqrt[p]{-u}$; if $u=0$, then we get $y=x=\sqrt[p]{z}$. Otherwise we put $\lambda=y / x$ and find the system

$$
\frac{\lambda^{2}-1}{\lambda}=\frac{u}{z}, \quad x^{p} \lambda=z .
$$

Here the rational function $\lambda-1 / \lambda$ is strictly increasing (its graphic consists of two lines) and the first equation in (2.3) for $u / z \neq 0, \infty$ has a solution $\lambda_{0} \neq 0$. Hence we get $x=\sqrt[p]{z / \lambda_{0}}, y=\lambda_{0} x$.

The map (2.1) has bounded right inverse (see Section 3.2 below). But when $p \geq 3$ is odd and $n \geq 3$, or when $p \geq 5$ is odd and $n=2$ then we can modify this example to such that $\left\{F^{-1}\right\}_{r}$ must be unbounded.

In the first case we put

$$
\begin{aligned}
\left(x_{1}, \ldots, x_{n-1}, y\right) & \\
& \mapsto\left(x_{1}^{p-1} y, x_{1}^{p-2}\left(y^{2}-x_{1}^{2}\right), x_{2}^{p-2}\left(y^{2}+x_{1}^{2}\right), \ldots, x_{n-1}^{p-2}\left(y^{2}+x_{1}^{2}\right)\right) .
\end{aligned}
$$

Then the first two equations $x_{1}^{p-1} y=z_{1}, x_{1}^{p-2}\left(y^{2}-x_{1}^{2}\right)=z_{2}$ are the same as in $(2.2)$ and admit a solution such that $\left(x_{1}, y\right) \neq(0,0)$. Thus $y^{2}+x_{1}^{2} \neq 0$ and the other equations can be easily solved. Therefore it remains to prove that the right inverse $H$ to $F$ is unbounded.

Take the following family $z_{\varepsilon}, \varepsilon>0, \varepsilon \rightarrow 0$ of points from the target space:

$$
z_{\varepsilon}=\left(0,-\varepsilon^{p}, 1,0, \ldots, 0\right) \text {. }
$$


The first equation $x_{1}^{p-1} y=0$ gives either $x_{1}=0$ (but then the second equation would mean that $0=-\varepsilon^{p}$ ), or $y=0$ (what we assume). Next, we get $x_{1}=\varepsilon$ and hence $y^{2}+x_{1}^{2}=\varepsilon^{2}$. Finally, we find $x_{2}=\varepsilon^{-2 /(p-2)}, x_{3}=\ldots=x_{n-1}=0$. We see that $H\left(z_{\varepsilon}\right)=\left(\varepsilon, \varepsilon^{-2 /(p-2)}, 0, \ldots, 0\right)$ and $\left|H\left(z_{\varepsilon}\right)\right| \rightarrow \infty$, whereas $\left|z_{\varepsilon}\right| \rightarrow 0$ as $\varepsilon \rightarrow 0$. This means that $H$ is unbounded.

The next example is a generalization of the example $(x, y) \mapsto\left(x^{2}\left(x^{3}-\right.\right.$ $\left.y^{3}\right), x^{3} y^{2}$ ) from the book [3]. Namely, we define

$$
(x, y) \stackrel{F}{\longmapsto}(z, u)=\left(x^{p-3}\left(x^{3}-y^{3}\right), x^{p-2} y^{2}\right) .
$$

If $z=0$, then we put $y=x$ and find $x^{p}=u$. If $u=0$, then we put $y=0$ and find $x^{p}=z$. If $(z, u) \neq(0,0)$, then with $\lambda=y / x$ we arrive to the system $\left(1-\lambda^{3}\right) / \lambda^{2}=z / u, x^{p} \lambda^{2}=u$. The graphic of the function $R(\lambda)=\lambda^{-2}-\lambda$ consists of two components (for $\lambda<0$ and for $\lambda>0$ ), where the right component has unbounded image $\left(\lim _{\lambda \rightarrow 0^{+}}=\infty\right.$ and $\left.\lim _{\lambda \rightarrow \infty}=-\infty\right)$ and $R(\lambda)>0$ in the left component. Thus always there exists a solution $(\lambda, x)$ such that $\lambda>0$ and $x=\sqrt[p]{u / \lambda^{2}}$. This gives the surjectivity.

To show absence of the Banach property, we take the following curve in the image: $z=1, u=-\varepsilon<0, \varepsilon \rightarrow 0$.

We have then $R(\lambda)=-1 / \varepsilon \rightarrow-\infty$. This implies that $\lambda \sim 1 / \varepsilon \rightarrow \infty$. Thus from the equation $x^{p} \lambda^{2}=u$ we find $x \sim \varepsilon^{3 / p}$. Finally $y=\lambda x \sim \varepsilon^{-1+3 / p} \rightarrow \infty$ (as $p \geq 5$ ). This means that $|(x, y)| \rightarrow \infty$. $\left\{F^{-1}\right\}_{r}$ is unbounded.

2.2. The case $p \geq 4$ even and $n \geq 2$. We write the source space $\mathbb{R}^{n}$ as $\mathbb{R}^{n-1} \times \mathbb{R}^{1}=\{(x, y)\}$ and define the map $F:(x, y) \mapsto z$ as follows

$$
\begin{aligned}
& z_{1}=x_{1}^{p-2}\left(y^{2}-x_{1}^{2}\right), \\
& z_{2}=x_{1}^{p-3} y\left(y^{2}-9 x_{1}^{2}\right), \\
& z_{3}=x_{2}^{p-1}\left(y-2 x_{1}\right), \\
& \ldots \ldots \ldots \ldots \cdots \\
& z_{n}=x_{n-1}^{p-1}\left(y-2 x_{1}\right) .
\end{aligned}
$$

Since $F(0, y)=0$, the map is not proper. The surjectivity we show firstly in the 2-dimensional case, i.e. we solve the system

$$
x^{p-2}\left(y^{2}-x^{2}\right)=z, \quad x^{p-3} y\left(y^{2}-9 x^{2}\right)=u
$$

(analogous to (2.2)). If $z=0$, then we get $y=x$ or $y=-x$ and $-8 x^{p}=u$ or $8 x^{p}=u$; one of the latter equations has a solution (recall that $p$ is even). If $u=0$, then we get $y=0$ or $y=3 x$ and $-x^{p}=z$ or $8 x^{p}=z$.

Note that here, when $(z, u) \neq(0,0)$, we have $y \neq 2 x$. If $z=u=0$, then $x=0$ and $y$ is arbitrary; in particular, we can choose $y \neq 2 x=0$. 
Assume that $z \neq 0 \neq u$. Putting $\lambda=y / x$ we arrive to the system

$$
\frac{\lambda\left(\lambda^{2}-9\right)}{\lambda^{2}-1}=\frac{u}{z}, \quad x^{p}\left(\lambda^{2}-1\right)=z
$$

(analogous to (2.3)). The rational function $R(\lambda) \stackrel{\text { df }}{=} \lambda\left(\lambda^{2}-9\right) /\left(\lambda^{2}-1\right)$ has zeroes and poles in alternate positions; thus $R$ is strictly increasing and its graphic consists of three pieces separated by the poles $\lambda= \pm 1$. Moreover, $R( \pm \infty)= \pm \infty$.

Thus the first equation in (2.7) for $u / z \neq 0, \infty$ has exactly three solutions $\lambda_{1}<\lambda_{2}<\lambda_{3}$ separated by the poles of $R(\lambda)$. We have $\lambda_{2}^{2}-1<0<\lambda_{1}^{2}-1$. This means that the second equation in (2.7) has a solution $x=\sqrt[p]{z /\left(\lambda_{i}^{2}-1\right)}$, $i=1$ or $i=2$. Next, we put $y=\lambda_{i} x$.

Note also that in this choice we have $\lambda_{1,2} \neq 2$ (they are $<1$ ), what means that $y \neq 2 x$.

Consider now the case $n>2$. Then the further equations $x_{2}^{p-1}\left(y-2 x_{1}\right)=$ $z_{3}, \ldots$ can be solved when $y-2 x_{1} \neq 0$. But $y$ and $x_{1}$ are obtained from the first two equations. We have shown before that these two equations always admit a solution such that $y \neq 2 x_{1}$.

Now we prove that the map (2.6) has only unbounded right inverses. Take the curve $\left\{z_{\varepsilon}\right\}_{\varepsilon>0}$ in the target given by $z_{1}=-\varepsilon, z_{2}=-\varepsilon^{3}, z_{3}=\ldots=z_{n}=0$ (i.e. we consider only the 2-dimensional case).

The system (2.7) takes the form $R(\lambda)=\varepsilon^{-2}, x_{1}^{p}\left(\lambda^{2}-1\right)=-\varepsilon$. We should have $\lambda^{2}-1<0$, i.e. we are in the middle component of the graphic of the function $R(\lambda)$. Moreover, as $\varepsilon \rightarrow 0$ we have $R(\lambda) \rightarrow+\infty$ and hence $\lambda \rightarrow 1^{-}$. More precisely, $\lambda=1-4 \varepsilon^{2}+\ldots$ and $\lambda^{2}-1 \sim-8 \varepsilon^{2}$. But then the second equation gives $x_{1} \sim(8 \varepsilon)^{-1 / p} \rightarrow \infty$ and $y \sim x \rightarrow \infty$.

We have then the unboundedness of $\left\{F^{-1}\right\}_{r}$.

2.3. The case $p=2$ and $n=4$. We represent $\mathbb{R}^{4}$ as $\mathbb{C}^{2}$, thus we introduce the coordinates $(x, y)=\left(x_{1}+i x_{2}, y_{1}+i y_{2}\right), i=\sqrt{-1}$ in the source space and $(z, u)=\left(z_{1}+i z_{2}, u_{1}+i u_{2}\right)$ in the target space. The map is given by

$$
(x, y) \mapsto\left(x y+\bar{x}^{2}, x \bar{y}\right) .
$$

It is clear that the plane $\mathbb{C} \simeq\{x=0\} \subset \mathbb{C}^{2}$ is mapped to the point $(z, u)=0$. Let $(z, u) \in \mathbb{C}^{2}$. We have to solve the system of equations

$$
x y+\bar{x}^{2}=z, x \bar{y}=u .
$$

We can assume here that

$$
(z, u) \neq(0,0)
$$

We shall show that:

- the system (2.9)-(2.10) has always a solution such that $x \neq 0$. 
Assuming $x \neq 0$ we calculate $y$ from the first equation in (2.9) and substitute it to the second equation. We get

$$
y=\frac{z \bar{x}-\bar{x}^{3}}{x \bar{x}}
$$

and the equation

$$
x^{3}-\bar{z} x+u \bar{x}=0 .
$$

We claim that:

- the equation (2.12) has always a nonzero solution whatever $z, u$ satisfying (2.10) are.

Introduce the following planar vector fields

$$
x \rightarrow V(x)=x^{3}-\bar{z} x+u \bar{x} \quad \text { and } \quad x \rightarrow W(x)=-\bar{z} x+u \bar{x} .
$$

We have to show that $V$ has a critical point different from $x=0$.

The field $W$ is linear with the critical point $x=0$. This critical point can be isolated (when the determinant $\operatorname{det} W_{*}$ of the corresponding matrix is nonzero), or non-isolated (it occurs when $|u|=|z|$ ).

In the first case the index at $x=0$ of $W$ equals signdet $W_{*}= \pm 1$ and the same index at $x=0$ has the vector field $V$. Take a circle $S_{R}^{1}=\{|x|=R\}$, where $R$ is large. Then the index of $V$ along $S_{R}^{1}$ (i.e. the increment of $\arg \left(x^{3}-\bar{z} x+u \bar{x}\right)$ along $S_{R}^{1}$ ) equal 3. Because the latter index equals the sum of indices at the critical points inside $S_{R}^{1}$, there must exist a critical point $x_{0} \neq 0$ inside $S_{R}^{1}$.

In the second case, i.e. $|z|=|u|$, we rotate the complex variable $x=x_{1}+i x_{2}$ and the vector field $V$ in such a way that the critical line for $W(x)$ becomes $x_{1}=0$ and $u=-z>0$. We get also $V(x)=a x^{3}+2 u x_{1}$ for some $a \in \mathbb{C} \backslash 0$. In the polar coordinates $x=r e^{i \varphi}$ the equation (2.12) now takes the form

$$
|a| r^{3} e^{i(\theta+3 \varphi)}=2 u r \cdot(-\cos \varphi) .
$$

We get

$$
\begin{aligned}
\varphi & =\frac{1}{3}(\pi-\theta+2 \pi k), \quad k=0,1,2, \cos \varphi>0, \\
\text { or } \varphi & =\frac{1}{3}(-\theta+2 \pi k), \quad k=0,1,2, \cos \varphi<0 .
\end{aligned}
$$

These are equations for $\varphi$, which always have a solution $\varphi_{0}$. Next, we calculate $r$ from the equation (2.13), i.e.

$$
r_{0}=\sqrt{2 u\left|\cos \varphi_{0}\right| /|a|}>0 .
$$

REMARK 1. Looking more carefully into the above proof one can see that there always exists a solution to the equation (2.12) such that

$$
C_{1} \sqrt{|(z, u)|} \leq|x| \leq C_{2} \sqrt{|(z, u)|}
$$


for some constants $C_{1,2}>0$. This implies that also the solution to (2.11) is bounded, i.e. $|y| \leq C_{3} \sqrt{|(z, u)|}$. Therefore the map (2.8) admits a bounded right inverse. Of course, this inverse cannot be continuous.

Since the further examples of surjective and non-proper maps (from Sections 2.4 and 2.5) use the map (2.8) as one of their components, also they enjoy the Banach property. The same holds for the map (2.16) from Section 2.6 below.

REMARK 2. The map (2.8) can be generalized to the case of $p>2$ and even. Namely, one puts

$$
(x, y) \mapsto\left(x^{p-1} y+\bar{x}^{p}, x^{p-1} \bar{y}\right) .
$$

The proof of its surjectivity is practically the same as in the case $p=2$.

REMARK 3. Another example of surjective and non-proper four-dimensional quadratic map is given by the formula

$$
\left(x_{1} y_{1}-x_{2} y_{2}-5 x_{2}^{2}, x_{2} y_{1}+x_{1} y_{2}, x_{2} y_{1}-4 x_{1} x_{2},-4 x_{2} y_{2}+9 x_{1}^{2}-19 x_{2}^{2}\right) .
$$

This was our first example of such a map. Our initial proof of its surjectivity used real methods and was rather involved. But one can prove it topologically, like for the map (2.8).

2.4. The case $p=2$ and even $n \geq 6$. If $n=2 k$ is even, then we represent $\mathbb{R}^{2 k}$ as $\mathbb{C}^{k-1} \times \mathbb{C}^{1}$ (in the source and in the target) and put

$$
\left(x_{1}, \ldots, x_{k-1}, y\right) \mapsto\left(x_{1} y+\bar{x}_{1}^{2}, x_{1} \bar{y}, x_{2}^{2}, \ldots, x_{k-1}^{2}\right) .
$$

This map is surjective, because the 4-dimensional map (2.8) from the previous section is surjective and the complex map $\zeta \mapsto \zeta^{2}$ is surjective.

2.5. The case $p=2$ and odd $n \geq 7$. Let us represent $\mathbb{R}^{3}$ as a subspace $\widetilde{\mathbb{H}}$ of the quaternion space $\mathbb{H}=\{a+b i+c j+d k\}$, where $i^{2}=j^{2}=k^{2}=-1$ and $i j=$ $-j i=k, j k=-k j=i, k i=-i k=j$. Namely we put $\widetilde{\mathbb{H}}=\left\{\zeta=\zeta_{1}+\zeta_{2} i+\zeta_{3} j\right\}$.

Standard calculations show that $\zeta^{2}=\zeta_{1}^{2}-\zeta_{2}^{2}-\zeta_{3}^{2}+2 \zeta_{1} \zeta_{2} i+2 \zeta_{1} \zeta_{2} j \in \widetilde{\mathbb{H}}$. Moreover, all the $\widetilde{\mathbb{H}}$ is in the image. This follows from the fact that the unit sphere in $\mathbb{H}$ is the connected Lie group $S U(2)$ with the group multiplication defined by the multiplication of corresponding quaternions.

Therefore the maps $\mathbb{C} \times \mathbb{C} \times \widetilde{\mathbb{H}} \times \mathbb{C}^{k-3} \mapsto C \times \mathbb{C} \times \widetilde{\mathbb{H}} \times \mathbb{C}^{k-3}$,

$$
\left(x, y, \zeta, t_{1}, \ldots, t_{k-3}\right) \mapsto\left(x y+\bar{x}^{2}, x \bar{y}, \zeta^{2}, t_{1}^{2}, \ldots, t_{k-3}^{2}\right)
$$

provide the new examples.

2.6. The case $p=2$ and $n=5$. We represent the source and target spaces as $\mathbb{R}^{3} \times \mathbb{R}^{2}$ with the coordinates $(x, y)=\left(x_{0}, x_{1}, x_{2}, y_{1}, y_{2}\right)$ and $(z, u)=$ $\left(z_{0}, z_{1}, z_{2}, u_{1}, u_{2}\right)$ respectively. We put

$$
\begin{aligned}
& z=A(x) y+Q(x), \\
& u=b(x) y-R(x),
\end{aligned}
$$


where

$$
\begin{gathered}
A(x)=\left[\begin{array}{cc}
26 x_{1}+106 x_{2} & 33 x_{1}-7 x_{2} \\
13 x_{1}+36 x_{2} & -9 x_{1}-12 x_{2} \\
-10 x_{1}+5 x_{2} & 20 x_{1}+4 x_{2}
\end{array}\right], \\
Q(x)=\left(\begin{array}{c}
-119 x_{0}^{2}+60 x_{0} x_{1}+20 x_{0} x_{2}-26 x_{1}^{2}-80 x_{1} x_{2}+14 x_{2}^{2} \\
30 x_{0} x_{1}-24 x_{0} x_{2}-30 x_{1}^{2}-6 x_{1} x_{2}+24 x_{2}^{2} \\
-10 x_{0} x_{1}+25 x_{0} x_{2}+10 x_{1}^{2}-15 x_{1} x_{2}-25 x_{2}^{2}
\end{array}\right), \\
b(x)=x_{0}+x_{1}+x_{2}, \quad R(x)=\left(\begin{array}{c}
x_{0}^{2}+x_{1}^{2} \\
x_{0}^{2}+2 x_{2}^{2}
\end{array}\right) .
\end{gathered}
$$

In order to show surjectivity of this map one eliminates $y$ from the last equation in (2.16), i.e. one puts $y=(u+R(x)) / b(x)$ into the first equation. One gets the equation

$$
C(z, u) x+\frac{17}{4} \operatorname{grad} f(x)=0
$$

where

$$
C(z, u)=\left[\begin{array}{ccc}
-z_{0} & -z_{0}+26 u_{1}+33 u_{2} & -z_{0}+106 u_{1}-7 u_{2} \\
-z_{1} & -z_{1}+13 u_{1}-9 u_{2} & -z_{1}+36 u_{1}-12 u_{2} \\
-z_{2} & -z_{2}-10 u_{1}+20 u_{2} & -z_{2}+5 u_{1}+4 u_{2}
\end{array}\right]
$$

and $\operatorname{grad} f(x)$ is the gradient of the function

$$
f(x)=x_{0}^{4}-\left(x_{1}^{2}-2 x_{0}^{2}\right)^{2}-\left(x_{2}^{2}-2 x_{0}^{2}\right)^{2} .
$$

When solving the equation (2.17), we treat it as an equation for critical points of the vector field

$$
x \rightarrow V(x)=C(z, u) x+\frac{17}{4} \operatorname{grad} f(x)=W(x)+\frac{17}{4} \operatorname{grad} f(x)
$$

(like in Section 2.3). Moreover one should look for critical points laying outside the hyperplane $b(x)=0$ (in order to be able to find $y$ next). We shall assume that $(z, u) \neq(0,0)$.

Lemma 2. The homogeneous cubic vector field grad $f(x)$ has isolated critical point at $x=0$ with index equal 7 .

Proof. We use some results from the real algebraic geometry. Let $f(x)$ be a real homogeneous polynomial of even degree $2 k$ on $\mathbb{R}^{3}$. It defines a real algebraic curve $C=\{f=0\}$ on the real projective plane $\mathbb{R} P^{2}$. Assume that this curve is smooth. $C$ divides the projective plane into two domains $D_{+}=\{f>0\}$ and $D_{-}=\{f<0\}$. G. N. Khimshiashvili ([6]) has proved the following formula

$$
\text { index } 0 \operatorname{grad} f(x)=\chi\left(D_{+}\right)-\chi\left(D_{-}\right)=2 \chi\left(D_{+}\right)-1,
$$

where $\chi$ denotes the Euler-Poincaré characteristic (see also [1] for its proof). 
We apply this formula to the function (2.18). Using the affine coordinates $v_{1,2}=x_{1,2} / x_{0}$ it reads as

$$
f=x_{0}^{4}\left[1-\left(v_{1}^{2}-2\right)^{2}-\left(v_{2}^{2}-2\right)^{2}\right] .
$$

In the variables $\left(v_{1}^{2}, v_{2}^{2}\right)$ the curve $f=0$ is a circle in the interior of the first quadrant. Therefore the curve $C \subset \mathbb{R}^{2} \subset \mathbb{R} P^{2}$ has four components (ovals) located in the four quadrants. The set $D_{+}$is the union of four discs in the interior of the four ovals. Since $\chi($ disc $)=1$, by $(2.19)$ the formula for index follows.

REMARK 4. The absolute value of the index can be interpreted also in terms of the ovals, i.e. connected components of the curve $C$. Each oval is a two-sided circle which divides the projective plane into two parts: the interior diffeomorphic to a disc and the exterior diffeomorphic to a Möbius band. We shall call an oval even if it lies in the interiors of even number of other ovals and we call it odd if it lies in the interiors of odd number of other ovals. For example, the usual circle $x_{1}^{2}+x_{2}^{2}=x_{0}^{2}$ is an even oval. Denote by $p$ the number of even ovals and by $m$ the number of odd ovals. Then we have $\left|\chi\left(D_{+}\right)-\chi\left(D_{-}\right)\right|=|2(p-m)-1|$.

In the case (2.18) all the four ovals are even and we get $|2(p-m)-1|=7$.

I. G. Petrovsky ([8]) has proved the estimate $|2(p-m)-1| \leq 3 k^{2}-3 k+1$, $k=(1 / 2) \operatorname{deg} C$ (the Petrovsky inequality). We see that the index is maximal in our example (2.18).

V. I. Arnold ([1]) has interpreted the left-hand side of the Petrovsky inequality as |index| and the right-hand side as a Hodge number of Steenbrink's mixed Hodge structure in cohomologies of the Milnor bundle associated to the complexification of $f$.

By Lemma 2 the map $x \mapsto V(x) /|V(x)|$ from a sphere $S_{R}=\{|x|=R\}$ of a large radius $R$ to the unit sphere $\mathbb{S}^{2}$ has degree 7 . Hence the sum of indices of critical points of $V(x)$ equals 7 .

One of these critical points is $x=0$. If $\operatorname{det} W_{*}=\operatorname{det} C(z, u) \neq 0$, then

$$
\operatorname{index}_{0} V=\operatorname{index}_{0} W=\operatorname{sign} \operatorname{det} C(z, u)= \pm 1 .
$$

Since $(z, u) \neq 0$, there are only two possible degenerations of $W$ at the critical point $x=0$ :

(i) $\operatorname{dim} \operatorname{ker} C(z, u)=1$,

(ii) $\operatorname{dim} \operatorname{ker} C(z, u)=2$.

The fields $W=W_{z, u}$ and $V=V_{z, u}$ depend on the parameters $(z, u) \in \mathbb{R}^{5} \backslash 0$. The point $x=0$ is degenerate for them when $(z, u)$ belongs to the bifurcational hypersurface $\Sigma=\{\operatorname{det} C(z, u)=0\}$. One can also see that the case (ii) occurs only when $u=0$. 
LEMma 3. In the case (i) we have ind $\operatorname{de}_{0} V= \pm 1$ and in the case (ii) we have index $\operatorname{mo}_{0} V= \pm 1, \pm 3$.

Proof. Consider the case (i). The fact that this index is the same as the index of a linear non-degenerate field can be explained by means of the bifurcation theory and geometrically.

Bifurcation theory arguments. Since $\operatorname{det} V_{*}(0)=\operatorname{det} W_{*}(0)$, at least one of the eigenvalues of the matrix $V_{*}(0)=C(z, u)$ is equal 0 . Its multiplicity can be 1 , or greater (we can have a Jordan cell). But in the second case we can apply an additional rotation to $V$ reducing the situation to one zero eigenvalue. This means that we make a change of the form $V(x) \rightarrow \widetilde{V}(x)=D \cdot V(x)$, where $D$ is a constant invertible matrix. For example, $\left(\begin{array}{ll}0 & 1 \\ 1 & 0\end{array}\right)\left(\begin{array}{ll}0 & 1 \\ 0 & 0\end{array}\right)=\left(\begin{array}{ll}0 & 0 \\ 0 & 1\end{array}\right)$. We can also assume that the other eigenvalues of $\widetilde{V}_{*}(0)$ are hyperbolic, i.e. with nonzero real parts, and non-resonant.

If $\lambda_{0}=0$ and $\operatorname{Re} \lambda_{1,2} \neq 0$, then the bifurcation theory ([2]) asserts that there is a local change of variables $\left(\mathbb{R}^{3}, 0\right) \ni x \mapsto(\xi, \eta) \in\left(\mathbb{R}^{1}, 0\right) \times\left(\mathbb{R}^{2}, 0\right)$ such that the system $\dot{x}=\widetilde{V}(x)$ takes the form

$$
\dot{\xi}=U(\xi), \dot{\eta}=M \eta
$$

where $M$ is a constant hyperbolic matrix; (it is the Shoshitaishvili reduction theorem). Moreover, since the vector field is odd $(\widetilde{V}(-x)=-\widetilde{V}(x))$, the change $x \mapsto(\xi, \eta)$ is odd and the 1-dimensional field $\dot{\xi}=U(\xi)$ is odd. In particular, $U(\xi)=a_{l} \xi^{2 l+1}+\ldots, a_{l} \neq 0$ for some $l<\infty$. But the index of $(2.20)$ at $(\xi, \eta)=0$ equals $\operatorname{sign} a_{l} \cdot \operatorname{sign} \operatorname{det} M= \pm 1$.

(When $l=1$ the corresponding bifurcation is called the pitchfork bifurcation and relies on arising of two symmetric critical points of $V$ from the origin).

Geometrical argument. Take a sphere $S_{\varepsilon}=\{|x|=\varepsilon\}$ with the center at $x=0$ and small radius $\varepsilon>0$ and look at its image under the maps $W$ and $V$. If $W=W_{z, u}$ is non-degenerate, then $W\left(S_{\varepsilon}\right)$ is an ellipsoid and $V\left(S_{\varepsilon}\right)$ is a small perturbation of this ellipsoid. In the case of degeneracy of the type (i) $\left(W=W_{z_{0}, u_{0}}\right.$ for $\left.\left(z_{0}, u_{0}\right) \in \Sigma\right)$ the set $W\left(S_{\varepsilon}\right)$ becomes an ellipse together with its interior in the plane $\mathcal{P}=W\left(\mathbb{R}^{3}\right)$. We fix the left and right sides of the surface $\mathcal{P}$ (coorientation). There are two points $p_{1,2}$ in the sphere $S_{\varepsilon}$ which are sent to 0 by $W$, they are the intersection points of $S_{\varepsilon}$ with the line ker $W_{*}$. Near each $p_{i}$ the map $W_{z_{0}, u_{0}} \mid\left(S_{\varepsilon}, p_{i}\right)$ is smooth and regular and takes values in the plane $\mathcal{P}$. The map $V \mid\left(S_{\varepsilon}, p_{i}\right)$ is a small perturbation of the map $W_{z_{0}, u_{0}} \mid\left(S_{\varepsilon}, p_{i}\right)$. Thus the local surface $V\left(\left(S_{\varepsilon}, p_{1}\right)\right)$ is smooth and "passes" the origin either on the left side of the plane $\mathcal{P}$ or on the right side. The other local surface $V \mid\left(S_{\varepsilon}, p_{2}\right)$ passes the origin on the opposite side of $\mathcal{P}$; (because $V$ is odd). Now it becomes clear that a typical vector $v \in \mathbb{S}^{2}$ close to the vector $v_{0}$ orthogonal to $\mathcal{P}$ has only one preimage under the map $S_{\varepsilon} \stackrel{\Psi}{\longmapsto} \mathbb{S}^{2}, \Psi(x)=V(x) /|V(x)|$. 
(By the way, in Section 2.3, instead of solving the equation (2.13) in the degenerate case, one could obtain the result by showing that index of $V$ at 0 is \pm 1 , in the same way as above.)

Consider the case (ii). As in the case (i) we take a change $V(x) \rightarrow \widetilde{V}(x)=$ $D \cdot V(x)$ such that $\widetilde{V}_{*}(0)=\operatorname{diag}(0,0, \lambda), \lambda>0$. Again the reduction theorem gives a local change $\left(\mathbb{R}^{3}, 0\right) \ni x \mapsto(\xi, \eta) \in\left(\mathbb{R}^{2}, 0\right) \times\left(\mathbb{R}^{1}, 0\right)$ such that

$$
\dot{\xi}=U(\xi), \quad \dot{\eta}=\lambda \eta
$$

and the 2-dimensional vector field $\dot{\xi}=U(\xi)$ is odd. In fact, we have

$$
U(\xi)=U_{3}(\xi)+U_{5}(\xi)+\ldots,
$$

where $U_{3}(\xi)$ is a homogeneous cubic vector field defined as follows. The plane $\mathcal{P}=\operatorname{ker} \widetilde{V}_{*}(0)$ is tangent to the center manifold (i.e. $\eta=0$ ). We restrict $\widetilde{V}$ to $\mathcal{P}$ and project the values of $\widetilde{V}$ to $\mathcal{P}$ along the line $\mathcal{L}=\operatorname{Im} \widetilde{V}_{*}(0)$ (tangent to the unstable manifold $\xi=0)$. We have $U_{3}=$ projection of $\left.(D \cdot(17 / 4) \operatorname{grad} f)\right|_{\mathcal{P}}$.

Notice firstly that $U_{3}(\xi) \not \equiv 0$. Otherwise the homogeneous cubic vector field $D \cdot \operatorname{grad} f(x)$ would send the plane $\mathcal{P}$ to the line $\mathcal{L}$. Since the latter field is odd, it should send a whole line in $\mathcal{P}$ to zero. But then 0 would not be an isolated critical point of grad $f(x)$.

When the 2-dimensional vector field $\xi \mapsto U_{3}(\xi)$ is typical, i.e. 0 is an isolated singularity, the $\operatorname{index}_{0} U_{3}(\xi)= \pm 1$, or $= \pm 3$.

In the non-typical case $U_{3}(\xi)$ has zeroes along: a single line (e.g. $U_{3}=\xi_{1} \cdot \xi^{2}$ for $\xi=\xi_{1}+\sqrt{-1} \xi_{2} \in \mathbb{C}$ ), along two distinct lines (e.g. $U_{3}=\xi_{1} \xi_{2} \cdot \bar{\xi}$ ) and along a double line (e.g. $\left.U_{3}=\xi_{1} \cdot(2 \xi-\bar{\xi})\right)$. In the non-typical cases one should take into account the higher order components $U_{5}(\xi), U_{7}(\xi), \ldots$ of the field $U(\xi)$. One looks at the image $U\left(S_{\varepsilon}^{1}\right)$ of the small circle $S_{\varepsilon}^{1}=\{|\xi|=\varepsilon\}$.

As in the geometrical analysis of the case (i) the situations with one simple critical line or two different critical lines for $U_{3}$ are "safe" in the sense that the curve $U_{3}\left(S_{\varepsilon}^{1}\right)$ is locally a smooth embedded curve with intersections of local branches as the only singularities. At 0 two or four local branches intersect themselves. The perturbed curve $U\left(S_{\varepsilon}^{1}\right)$ also has smooth local branches near 0 (but avoiding 0 ). Using the fact that $U(\xi)$ is odd, one can easily see that the curve $U\left(S_{\varepsilon}^{1}\right)$ turns around the origin at most three times (in the clockwise or counter clockwise direction). Thus index ${ }_{0} U(\xi)$ equals to the index of some typical homogeneous cubic vector field.

The case with double critical line needs a separate investigation. It is rather easy to see that the curve $U_{3}\left(S_{\varepsilon}^{1}\right)$ has cusp-type singularities with vertices at the origin (e.g. $v_{2}^{2} \approx v_{1}^{3}$ ). There are two such cusps and the curve $U_{3}\left(S_{\varepsilon}^{1}\right)$ has no other self-intersections. The perturbed curve $U\left(S_{\varepsilon}^{1}\right)$ can be: either smooth, or 
with two cusps, or with two small loops near 0. As in the previous cases we see that the curve $U\left(S_{\varepsilon}^{1}\right)$ turns around the origin at most three times.

We have then $\operatorname{index}_{0} U(\xi)= \pm 1, \pm 3$. This together with (2.21) completes the proof of Lemma 3 .

Besides $x=0$ there can be some other obvious singular points of the vector field $V(x)$. Consider the equation $u=b(x) y-R(x)$ (see (2.16)) restricted to the hyperplane $b(x)=x_{0}+x_{1}+x_{2}=0$, i.e.

$$
\left.R(x)\right|_{b(x)=0}=-u \text {. }
$$

Any solution $\left(x_{1}^{(i)}, x_{2}^{(i)}\right)$ to $(2.22)$ gives a point $x^{(i)}=\left(-x_{1}^{(i)}-x_{2}^{(i)}, x_{1}^{(i)}, x_{2}^{(i)}\right)$ which is a solution to $(2.17)$; (here $i=1,2,3,4)$.

\section{LEMMA 4.}

(a) The only critical points $x \neq 0$ of the vector field $V(x)$ which lie on the hyperplane $b(x)=0$ are of the type $x^{(i)}$ defined by solutions to (2.22).

(b) The sum of the indices ind $\operatorname{ex}_{x^{(i)}} V$ over such points $x^{(i)}$ equals 0.

Proof. (i) The system (2.17) can be written in the form

$$
A(x)(u+R(x))+b(x)(Q(x)-z)=0 .
$$

From this it is seen why the points $x^{(i)}$ solve it.

Assume that $x \neq 0$ and $b(x)=0$. We have to show that then necessarily $u+R(x)=0$. But for this it is enough to show that the $3 \times 2$ matrix $A(x)=$ $A\left(x_{1}, x_{2}\right)$ has rank equal 2 when $\left(x_{1}, x_{2}\right) \neq 0$. Standard calculations show that the determinant of the first two rows of $A\left(x_{1}, x_{2}\right)$ vanishes along the lines $x_{1} \approx$ $-3.1 x_{2}$ and $x_{1} \approx-0.5 x_{2}$ and that the determinant of the last two rows vanishes along the lines $x_{1} \approx-3.8 x_{2}$ and $x_{1} \approx-0.3 x_{2}$.

(ii) The equation (2.22) takes form of the system

$$
\left(x_{1}+x_{2}\right)^{2}+x_{1}^{2}=-u_{1},\left(x_{1}+x_{2}\right)^{2}+2 x_{2}^{2}=-u_{2} .
$$

Typically it has 0 or 4 solutions. Moreover, the solutions disappear pairwise and simultaneously (two opposite pairs at the same moment). The corresponding critical points $x^{(i)}=x^{(i)}(u)$ of the field $V$ also disappear pairwise, in the socalled saddle-node bifurcation. If $x^{(i)}(u)$ and $x^{(j)}(u)$ meet for some $u=u_{0}$ (and next disappear) then $\operatorname{index}_{x^{(i)}} V+\operatorname{index}_{x^{(j)}} V=0$. This follows from calculation of the degree of the map $x \mapsto V(x) /|V(x)|$ restricted to a small sphere with center at $x^{(i)}\left(u_{0}\right)=x^{(j)}\left(u_{0}\right)$.

From his the equality $\sum \operatorname{index}_{x^{(i)}} V=0$ follows. 
Now we are able to complete the proof of surjectivity of the map (2.16). By Lemmas 2, 3 and 4 we have

$$
7=\operatorname{index}_{0} V+\sum \operatorname{index}_{x^{(i)}} V+\sum_{x: b(x) \neq 0} \operatorname{index}_{x} V,
$$

where the first summand takes one of the values $\pm 1, \pm 3$ and the second summand is zero. Thus the third summand is nonzero, what implies that there exists a point $x_{*}$ such that $b\left(x_{*}\right) \neq 0$ and $V\left(x_{*}\right)=0$. Then $(x, y)=\left(x_{*},\left(u+R\left(x_{*}\right) / b\left(x_{*}\right)\right)\right.$ is the solution to $(2.16)$.

REMARK 5. Before arriving to the example (2.16) (leading eventually to the equation (2.17)) we tried an example like in (2.16), but with $A(x)$ and $C(x)$ chosen in such a way that the elimination of $y$ leads to the equation $\widetilde{C}(z, u)+$ $17 x^{3}=0$, where $x=x_{0}+i x_{1}+j x_{2}$ is treated as a quaternion. We have not succeeded in solving it, because the vector field $x \mapsto x^{3}$ has index 1 .

But by the way we have discovered the following generalized Euler formula

$$
e^{i \varphi_{1}+j \varphi_{2}+k \varphi_{3}}=\cos \sqrt{\varphi_{1}^{2}+\varphi_{2}^{2}+\varphi_{3}^{2}}+\frac{i \varphi_{1}+j \varphi_{2}+k \varphi_{3}}{\sqrt{\varphi_{1}^{2}+\varphi_{2}^{2}+\varphi_{3}^{2}}} \sin \sqrt{\varphi_{1}^{2}+\varphi_{2}^{2}+\varphi_{3}^{2}} .
$$

When one writes $i \varphi_{1}+j \varphi_{2}+k \varphi_{3}=\mathbf{i} \rho$, where $\rho=\sqrt{\varphi_{1}^{2}+\varphi_{2}^{2}+\varphi_{3}^{2}}$ and $\mathbf{i}=$ $\left(i \varphi_{1}+j \varphi_{2}+k \varphi_{3}\right) / \sqrt{\varphi_{1}^{2}+\varphi_{2}^{2}+\varphi_{3}^{2}}$ is a unit imaginary quaternion (which satisfies $\mathbf{i}^{2}=-1$ ), then this formula reads as $e^{\mathbf{i} \rho}=\cos \rho+\mathbf{i} \sin \rho$ and becomes obvious.

In particular, we have the following pretty identities

$$
\begin{array}{rlrl}
e^{\mathbf{i} m \rho} & =\left(e^{\mathbf{i} \rho}\right)^{m}, & & m \in \mathbb{Z}, \\
e^{\mathbf{i}(\rho+2 \pi)} & =e^{\mathbf{i} \rho}, & \\
e^{\mathbf{i}(\rho+\pi)} & =e^{(-\mathbf{i})(\pi-\rho)}=-e^{\mathbf{i} \rho}, & & 0 \leq \rho \leq \pi .
\end{array}
$$

\section{Properness of surjective maps in low dimensions}

Proof of The Point (b) of Theorem 1 . When $n=1$ any homogeneous map is of the form $x \mapsto a x^{p}$. It is surjective if and only if $p$ is odd and $a \neq 0$. It is proper if and only if $a \neq 0$. Therefore the implication: surjective $\Rightarrow$ proper is true.

When $p=1$ the map is linear and the equality $\operatorname{dim} \operatorname{ker} F=\operatorname{dim} \operatorname{coker} F$ shows the equivalence: surjective $\Leftrightarrow$ proper.

Now we give a short proof of the theorem of Izmailov and Tret'yakov ([4]) which states that for $n=2,3$ a homogeneous quadratic map $F: \mathbb{R}^{n} \mapsto \mathbb{R}^{n}$ with nontrivial kernel cannot be surjective. Our proof is different than in [4].

We denote $\mathbb{R}^{n}=\mathbb{R}^{n-k} \times \mathbb{R}^{k}=\{(x, y)\}$ such that the surface $\{0\} \times \mathbb{R}^{k}=$ $\{x=0\}$ lies in $\operatorname{ker}^{2} F$; thus $F(0, y)=0$. We can assume that $0<k<n$. 
If $n=2$, then $k=1$ and, after applying a linear change in the image, we get one of the two situations:

$$
F=\left(a x^{2}, x y\right) \quad \text { or } \quad F=F(x)=\left(a x^{2}, b x^{2}\right) .
$$

Since the first component $a x^{2}$ takes values in $a \mathbb{R}_{+}$(where $\mathbb{R}_{+}=\{t \in \mathbb{R}: t \geq 0\}$ ), the map cannot be surjective.

If $n=3, k=2$, then we have one of the three possibilities (modulo invertible changes): $\left(a x^{2}, x y_{1}, x y_{2}\right),\left(a x^{2}, b x^{2}, x y_{1}\right),\left(a x^{2}, b x^{2}, c x^{2}\right)$. Here also the first component takes values in $a \mathbb{R}_{+}$.

Let $n=3, k=1$ and $(x, y)=\left(x_{1}, x_{2}, y\right)$. Then, besides the case $F=F(x)$ (when $\operatorname{Im} F$ is at most 2-dimensional), we have one of the two cases:

(i) $\left(x_{1} y+a x_{2}^{2}, x_{2}\left(y+b x_{1}+c x_{2}\right)+d x_{1}^{2}, f_{3}(x)\right)$,

(ii) $\left(x_{1} y+a x_{2}^{2}, f_{2}(x), f_{3}(x)\right)$.

If the form $f_{3}$ in the case (i) is (semi-)definite, then $\operatorname{Im} F \neq \mathbb{R}^{3}$. Otherwise, we can assume that $f_{3}=x_{1} x_{2}$. Assuming $z_{3}=f_{3}(x, y)=0$, we get $\left.\operatorname{Im}\left(f_{1}, f_{2}\right)\right|_{f_{3}=0}=\left(a \mathbb{R}_{+} \times \mathbb{R}\right) \cup\left(\mathbb{R} \times d \mathbb{R}_{+}\right) \neq \mathbb{R}^{2}$.

In the case (ii) we firstly exclude the cases when $f_{2}$ or $f_{3}$ is (semi-)definite. There remain the three subcases:

$$
\begin{gathered}
F=x_{1} G(x, y), \\
\left(x_{1} y+x_{2}^{2}, x_{1}\left(a x_{1}+b x_{2}\right), x_{1}\left(c x_{1}+d x_{2}\right)\right), \\
\left(x_{1} y,\left(x_{2}-a x_{1}\right)\left(x_{2}-b x_{1}\right), x_{1}\left(c x_{1}+d x_{2}\right)\right) .
\end{gathered}
$$

The first subcase is practically the same as the case $n=3, k=2$. In the second subcase, assuming $z_{3}=0$, we get the image of $\left.\left(f_{1}, f_{2}\right)\right|_{f_{3}=0}$ in a set $\mathbb{R} \times \alpha \mathbb{R}_{+}$for some constant $\alpha$ (depending on $\left.a, b, c, d\right)$. In the third subcase, the restriction $z_{3}=0$ give the image of $\left.\left(f_{1}, f_{2}\right)\right|_{f_{3}=0}$ in a set $\left(\{0\} \times \alpha \mathbb{R}_{+}\right) \cup\left(\mathbb{R} \times \beta \mathbb{R}_{+}\right)$ for some constants $\alpha, \beta$.

REMARK 6 . The reader can observe that in all situations considered in this section the number of monomials $x_{i} y_{j}$ is smaller than the dimension of the space $X=\mathbb{R}^{n}, k(n-k)<n$.

In an analogous way we can treat the case $p=2, n=5$ and $k=4$.

Proof of the point (b) of Theorem 2. In view of Theorem 1(b) it is enough to show that homogeneous cubic surjective maps of $\mathbb{R}^{2}$ always admit bounded right inverses.

By Lemma 1 we can assume that $F$ is not proper, i.e. that it is of the form

$$
z=x^{3} f(y / x), u=x^{3} g(y / x),
$$

where $f(\lambda)$ and $g(\lambda)$ are polynomials of degree $\leq 2$. These polynomials cannot have degrees $\leq 1$, because one can reduce such map to the following "contraction 
map" $(x, y) \mapsto\left(x^{3}, x^{2} y\right)$ (whose image does not contain the points $\left.(0, u), u \neq 0\right)$. By the same reason the situation when the polynomials $f$ and $g$ have a common linear factor (maybe over the field $\mathbb{C}$ ) is not admissible.

So, assume that $R(\lambda)=g(\lambda) / f(\lambda): \mathbb{R} P^{1} \mapsto \mathbb{R} P^{1}$ is a rational function of degree 2 with two zeroes and two poles; maybe some zero or pole lies at infinity. Using the Möbius transformations $\lambda \mapsto a \lambda+b$, which fixes $\lambda=\infty$ and arise from linear transformations $y \mapsto a y+b x$, and applying some linear transformations in the $(z, u)$-plane we reduce the situation to such that the poles are at $\lambda=0$ and $\lambda=\infty$ and the zeroes are at $\lambda=1$ and at $\lambda=c \neq 0, \infty$. Thus $R(\lambda)=$ $(\lambda-1)(\lambda-c) / \lambda$.

We claim that if $c>0$, then the map $F$ is not surjective. To see this, consider the equation $R(\lambda)=d$. This equation takes the form $\lambda^{2}-(c+d+1) \lambda+c=0$. Its discriminant equals $(c+d+1)^{2}-4 c$ and is negative for $d=-c-1$.

If $c<0$, then the situation is the same as in the case of the system (2.2). The corresponding system $x^{2} y=z, x(y-x)(y-c x)=u$ has "bounded" solutions for $z=0$ and for $u=0$. These bounded solutions are prolonged to bounded solutions when $z / u$ is small and when $u / z$ is small. (When $z / u$ is small then we have the system $\lambda /(\lambda-1)(\lambda-c)=z / u, x^{3}(\lambda-1)(\lambda-c)=u$ with the solution $\lambda \approx c \cdot z / u, x \approx \sqrt[3]{u / c})$.

Now we solve the system $R(\lambda)=d, x^{3} \lambda=z$, where $d=u / z$ takes values separated from zero and infinity. So the first equation admits a solution $\lambda_{0}$ uniformly separated from zero and from infinity. Therefore the second equation has the solution $x=\sqrt[3]{z / \lambda_{0}}$ with the bound $|x| \leq$ const $\cdot|z|$. Also $|y|=\left|\lambda_{0} x\right| \leq$ const $\cdot|z|$.

\section{REFERENCES}

[1] V. I. ARNold, Index of a singular point of a vector field, the Petrovsky-Oleinik inequalities and mixed Hodge structures, Funktsional. Anal. i Prilozhen. 12 (1978), 1-14. (Russian)

[2] Geometrical Methods in the Theory of Ordinary Differential Equations, Springer-Verlag, New York-Berlin, 1983; Nauka, Moscow, 1978. (Russian)

[3] A. F. Izmailov and A. A. Tret'yakov, Factor Analysis of Nonlinear Mappings, Nauka, Moscow, 1994. (Russian)

[4] On existence of solutions of the Cauchy problem for a partial differential equation of first order in the degenerate case, Differential Equations 37 (2001), in press. (Russian)

[5] The problem of invertibility of quadratic mappings, preprint (2000), Moscow State University. (Russian)

[6] L. L. Dines, On the mapping of quadratic forms, Bull. Amer. Math. Soc. 47 (1941), 494-498.

[7] G. N. Khimshiashvili, On local degree of a smooth mapping, Soobshch. Akad. Nauk Gruzii 85 (1977), 309-311. (Russian) 
[8] I. G. Petrovsky, On the topology of real algebraic curves, Ann. Math. 39 (1938), $187-209$.

[9] A. A. Tret'yakov, The implicit function theorem in degenerate problems, Russian Math. Surveys 42 (1987), 179-180; Uspekhi Mat. Nauk 42 (1987), 215-216. (Russian)

Manuscript received November 11, 2001

Aleksey Tret'yakov

Institute of Mathematics and Physics

Academy of Podlasie

Orlicz-Dreszera 19/21

08-110 Siedlce, POLAND

E-mail address: tret@ap.siedlce.pl

HENRYK ŻOŁĄDEK

Institute of Mathematics

Warsaw University

Banacha 2

02-097 Warsaw, POLAND

E-mail address: zoladek@mimuw.edu.pl

TMNA : Volume $19-2002-\mathrm{N}^{\circ} 2$ 